\title{
HBT and Initial Conditions for Hydrodynamic Expansion in A+A Collisions
}

\author{
M. Gyulassy ${ }^{1}$, Iu. Karpenko ${ }^{2}$, A. V. Nazarenko ${ }^{2}$, and Yu. M. Sinyukov ${ }^{2}$ \\ ${ }_{1}$ Columbia University 538 West 120th St New York, NY 10027, USA \\ ${ }^{2}$ Bogolyubov Institute for Theoretical Physics, 14-b Metrologichna Str, Kiev 143,03680, Ukraine
}

Received on 16 January, 2007

\begin{abstract}
The behavior of interferometry radii in central A+A collisions at different energies and also for different nuclei or impact parameters indicates initial transverse flow at a very early stage of the matter evolution. Development of such flow at the pre-thermal partonic stage is considered.
\end{abstract}

Keywords: Heavy ion collisions; Partons; Initial conditions; Hydrodynamics

\section{INTRODUCTION}

The first results of femtoscopy, or HBT, analysis of RHIC experiments [1] (as it was first announced by the STAR Collaboration) have revealed unexpected results - the so-called RHIC HBT puzzle [2]. The puzzle implies, firstly, that the absolute values of the interferometry radii/volume in central $\mathrm{Au}+\mathrm{Au}$ collisions do not change essentially at RHIC as compared to SPS energies for $\mathrm{Pb}+\mathrm{Pb}$ collisions despite much higher multiplicities. It was in contrast with, expected at that time, the possibility of the proportionality law between the interferometry volumes and multiplicities. At the same time there is an approximate proportionality between the interferometry volume and different initial volumes which can be associated, e.g., with the number of participants (nucleons of nuclei) in the collision process and, thus, with the multiplicity. Secondly, the ratio of outward to sideward transverse radii is opposite to what was expected in standard hydrodynamic and hadronic cascade pictures. The ratio measured by the STAR and PHENIX collaborations at RHIC BNL is close to unity in a wide momentum region. At first sight these observations are in a contradiction with an existence of quark-gluon plasma and a mixed phase as it implies long-lived pion radiation which usually results in the large ratio of outward to sideward transversal radii. As a result, the phenomenological parameterizations, like the blast wave model, just ignore emission from the surface of the expanding system despite the fact that it should last at least about the extracted life-time of the fireball: $10-12 \mathrm{fm} / \mathrm{c}$.

These notes represent the possible explanation of the peculiarities of the observed behavior of the interferometry radii based on an analysis of the temporal evolution of observables $[3,4]$. As a result, one can conclude that initial flows in prethermal partonic matter, which precede hydrodynamical expansion, should develop in the system. We discuss the possible scenario of the pre-thermal evolution of partonic matter and estimate the collective velocities at this early stage of the processes of ultrarelativistic A+A collisions.

\section{ANALYSIS AND TREATMENT OF EXPERIMENTAL DATA}

As was shown in Ref.[3] the phase-space density of thermal pions totally averaged over freeze-out hypersurface $\sigma$ and over momenta, except the longitudinal one (rapidity is fixed, e.g., $y=0),\langle f\rangle$, is an approximate integral of motion.

The conservation of the average phase space density (APSD) allows one to study the hadronization stage of the matter's evolution based on the possibility to define the APSD of thermal pions at the final stage of the evolution through the observed spectra and interferometry volumes [3]. The results for the pion APSD at the AGS, SPS, and RHIC demonstrate the violation of the regime of monotonic increase of this quantity with energy: a plateau starts at low SPS energies. It indicates, apparently, a transformation of an excess of growing initial energy to non-hadronic forms of matter which starts at that energy interval. A saturation of the APSD at RHIC energies can be treated as an existence of the limiting Hagedorn temperature of hadronic matter, or maximal temperature of deconfinement [4].

Let us use these results for an analysis of the behavior of the pion interferometry volumes $V_{\text {int }}$. If one considers them at small transverse momenta, then they can be represented $a p$ proximately through the APSD as the following:

$$
V_{i n t} \simeq C \frac{d N / d y}{\langle f\rangle T_{e f f}^{3}}
$$

It is easy to see then that at any fixed energy $\sqrt{s_{N N}}, V_{\text {int }}$ is nearly constant in time since the values $d N / d y$, the APSD $\langle f\rangle$ and the effective temperature $T_{\text {eff }}$ in r.h.s. of Eq. (1) are approximately conserved for thermal pions during the chemically frozen hydro-evolution. As a result, the HBT microscope at diverse energies "measures" the radii that are similar to the sizes of colliding nuclei. It explains the experimental observations that at the same collision energy, $V_{\text {int }}$ depends strongly on the sizes of colliding nuclei and on the impact parameters in non-central collisions [5].

RHIC experiments show clearly that there is no proportionality law between $V_{\text {int }}$ and $d N^{\pi} / d y$ : the latter value grows with energy significantly faster than $V_{i n t}$. This fact is the main component of the HBT puzzle. According to Eq. (1), a proportionality between $V_{\text {int }}$ and the particle numbers $d N / d y$ may be destroyed by a factor $\langle f\rangle T_{e f f}^{3}$. So, if the APSD and $V_{i n t}$ only slightly grow with energy, mostly an increase of $T_{\text {eff }}^{3}$ could compensate a growth of $d N / d y$ in Eq. (1). One can see that it is the case: for example, the ratio of the cube of effective temperatures of negative pions at $\sqrt{s_{N N}}=200 \mathrm{GeV}$ (RHIC) to one 


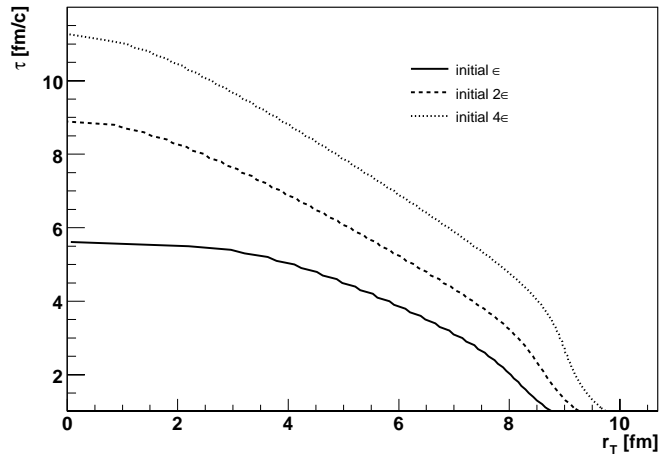

FIG. 1: The typical freeze-out hypersurfaces with the fixed f.o. energy density presented in $\tau-r$ plane for Bjorken-like azimuthally symmetric hydrodynamic expansion with equation of state $P=\varepsilon / 3$ and zero initial transverse flow. The curves correspond to the different initial energy densities $\varepsilon_{i}\left(\tau_{i}, y, r\right)=\varepsilon_{0}\left(\tau_{i}, y, r\right), 2 \varepsilon_{0}\left(\tau_{i}, y, r\right)$, $4 \varepsilon_{0}\left(\tau_{i}, y, r\right)$ distributed in $r$-plane according to the Woods-Saxon formula. The initial proper time is $\tau_{i}=1 \mathrm{fm} / \mathrm{c}$.

at $40 \mathrm{AGeV}$ (CERN SPS) gives approximately 2, while the ratio of the corresponding mid-rapidity densities is approximately equal to 3 . It can be so only if accompanied by an increase of the pion transverse flow in $\mathrm{A}+\mathrm{A}$ collisions with energy. If the intensity of flow grows, it leads to a reduction of the corresponding homogeneity lengths which contribute to the interferometry radii. This effect can almost compensate a contribution to observed interferometry volumes of the geometrical system sizes that grow with energy. The question is then: why does the intensity of flow grow? It is clear that an increase of collision energy $\sqrt{s}$ results in a rise of initial energy density $\varepsilon$ and hence of (maximal) initial pressure $p_{\max }$. At the same time the initial transverse acceleration $a=\operatorname{grad}(p) / \varepsilon \propto p_{\max } / \varepsilon$ does not change. Thus, one can conclude that there could be the two reasons for an increase of transverse pion flows with collision energy. The first one is obvious, it is an increase of the duration of the hydroevolution that the system needs to reach the same (or less) freeze-out energy density or temperature at higher initial density (see Fig. 1)

However, apparently, the relativistic hydrodynamic picture overestimates the increase of the longitudinal interferometry radii, that is associated with the life-time of the system, as compared to the experimental data.

The other reason for an increase of the observed transverse flow is the presence of the initial transverse velocity which may develop at the pre-thermal partonic stage and obviously has an influence on the evolution and the intensity of transverse flow at freeze-out. Moreover, what is essentially important, this factor has the direct connection to the second component of the HBT puzzle: the unexpectedly small ratio of outward to sideward interferometry radii. In relativistic hydrodynamics or realistic hydro-inspired parametrizations, the freeze-out hypersurface should be enclosed, so the protractive surface emission of pions (hadrons) from fairly cold periphery of the expanding system takes place. Normally, it should lead

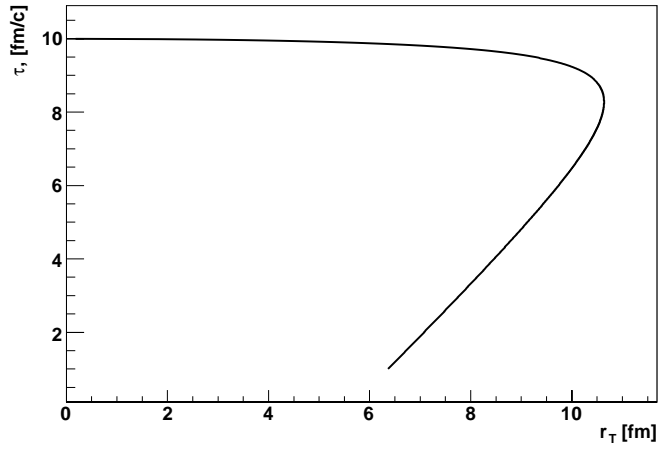

FIG. 2: The dynamical realization of the freeze-out with positive $r-\tau$ correlations at constant energy density [7] based on the (3+1)D exact analytical solutions of relativistic hydrodynamics [8] with intensive initial transverse flows.

to large $R_{\text {out }}$ to $R_{\text {side }}$ ratio, however, as demonstrated in Ref. [6], it is possible, nevertheless, to describe the data successfully, including $R_{\text {out }}$ to $R_{\text {side }}$ ratio, if there are positive $r-t$ correlations between the radial $r$ coordinates and times $t$ of surface emission of the particles. The term associated with these correlations gives the negative contribution to $R_{\text {out }}$ interferometry radius and so compensates the positive contribution to it caused by long-lived surface emission.

Only the fit with positive $r-t$ correlations, as presented in Fig.2, results in a good description of the spectra for pions, kaons and protons and of pion interferometry data, including $R_{\text {side }}$ and $R_{\text {out }}$. All details are presented in Ref.[6].

One of the most important observations is that the r-t correlation at the freeze-out hypersurface, according to equations of relativistic hydrodynamics, can be predominantly positive if the system has at the initial moment developed transverse flow[17]. The typical situation is presented in Fig.1 and Fig.2. The former figure corresponds to an absence of the initial transverse flow, the second describes an intensive blast-like expansion into vacuum that starts at the early stage of the evolution, say, at $\tau=1 \mathrm{fm} / \mathrm{c}$. In the first case the negative $r-t$ correlations between the surface emission points takes place, and it leads to a positive contribution to $R_{\text {out }}$ in addition to a large positive contribution associated with the protracted surface emission. In the second case the latter positive term is compensated by the negative one arising from positive $r-t$ correlations. It leads to experimentally observed $R_{\text {out }}$ to $R_{\text {side }}$ ratio in presence of protracted surface emission [6].

\section{PRE-THERMAL PARTONIC STAGE: THE FREE-STREAMING APPROXIMATION}

The problem of the formation of transverse velocity at pre-thermal partonic stage leads inevitably to considering the complex matter of the initial stage in ultrarelativistic A+A collisions and the problem of thermalization. In these notes we will not discuss in detail this very complicated topic, keeping in mind a quite simple physical picture and apply it phenom- 
enologically.

Let us imagine a box (with size $L$ ) that has ideally reflecting walls and contains standing (electromagnetic) waves inside. Then collide the two such boxes with sufficient energy to crush them completely. Standing waves then will be destroyed due to a stochastization that is accompanied by the crashing processes [10]. In other words, strong correlations between phases of travelling "backward" and "forward" waves, with discrete momenta, say, $2 \pi / L$ and $-2 \pi / L$, caused by ideal reflections from the opposite walls, will vanish and instead the random phases $\exp \left(\alpha_{p_{i}}\right)$ will appear:

$\sin \frac{2 \pi x}{L}=\frac{1}{2}\left(\exp \frac{i 2 \pi x_{\mathrm{T}}}{L}-\exp \frac{-i 2 \pi x_{\mathrm{T}}}{L}\right) \Rightarrow \sum \rho_{p_{i}} \mathrm{e}^{\alpha_{p_{i}}} \mathrm{e}^{i p_{i} x_{T}}$.

In the case of very weak field we will see then, say, two incoherent photons travelling, for instance, in the transversal plane in opposite directions.

Let us provide an analogy now with high energy nucleusnucleus collisions by imaging them as the collisions of the two "boxes" (containing many "small boxes" - nucleons). Due to the non-commutativity of the gluon number operator with the operator of Lorenz boost, there is a huge number of coherent partons in the fast moving box - this state probably can be represent within the Color Glass Condensate (CGC) approach [11]. Correspondingly, after the collision there will be not just two gluons but the classical color field (because of large occupation number) expanding into vacuum. When the occupation number reduces, one can see the picture of the expanding system of incoherent partons. It may be called a "partonic explosion" when many hidden degrees of freedom, associated with incoherent partons with significant transverse momentum, are liberated almost suddenly. An estimate of the thermalization time for this system is a rather complicated problem and we just mention it later. It seems that partons interact weakly enough and that instability mechanisms [12] works not sufficiently fast as is necessary to reach momentum symmetrization (thermalization?) in the very small time, less than $1 \mathrm{fm} / \mathrm{c}$, required by hydrodynamics models to describe elliptic flows.

Let us simplify the problem again and consider now the developing of transverse velocity at the pre-thermal partonic stage in an approximation of free streaming evolution for these weakly interacting particles.

We start from the simple non-relativistic example. Let us assume the initial momentum distribution of particles with mass $m$ to be a spherically symmetric Gaussian with the width corresponding to a thermal Boltzmann distribution with uniform temperature $T_{0}$, no flows: $\mathbf{u}(t=0, \mathbf{r})=0$, and also assume a spherically symmetric Gaussian profile (with radius $R_{0}$ ) for the particle density. We then allow the particles to free stream. Then according to [3] the collective velocities, which can be defined at any time $t$ according to Eckart, become:

$$
u^{i}=\int \frac{d^{3} p}{m^{4}} p^{i} f(t, \mathbf{x} ; p)
$$

are

$$
\mathbf{u}(t, \mathbf{r})=\mathbf{r} \frac{t T_{0}}{m R_{0}^{2}+T_{0} t^{2}}
$$

As one can see, the collective velocities in the free streaming system grow with decreasing particle mass, and grow with the initial parameter $T_{0}$ for $m \neq 0$, and are independent of the initial "temperature" at $m=0$. Qualitatively, the same happens for a relativistic partonic gas.

Let us consider the relativistic partonic picture with the initial momentum distribution at Björken proper time $\tau=1 \mathrm{fm} / \mathrm{c}$ corresponding to "transverse momentum" Fourier components in the color field in the CGC picture found in Ref.[13]. Suppose that after the collision the similar transverse spectrum will appear for incoherent partons. As for the longitudinal ones we will use the local 3D isotropic quasi-thermal distribution as it was proposed in Ref.[14] based on the Schwinger mechanism of the partonic production: the partons created by a pulse of the strong chromo-electric field during collision process are distributed (locally) quite isotropically since the action of the field is limited in time. Let us use the boost-invariant approximation in mid-rapidity and the WoodsSaxon initial profile for energy density in transverse plane. Then the partonic distribution function at the initial proper time $\tau=\tau_{0}$ is:

$$
f_{0}=\frac{1}{\exp \frac{m_{T}}{T} \cosh \theta-1} \frac{1}{\exp \frac{1}{\delta}\left(r_{T}-R\right)+1},
$$

where $\theta$ is the difference between particle and fluid rapidities. The main parameters of the distribution agree with Refs.[13, 14]: $T=0.465 \Lambda_{s}, \delta=0.67 \mathrm{fm}, \Lambda_{s}=1.3 \mathrm{GeV}, \tau_{0}=1 \mathrm{fm} / \mathrm{c}$, $R=7.3 \mathrm{fm}$, and the partonic mass is taken to be equal to $m=$ $m_{0}=0.0358 \Lambda_{s}$. The evolution of this function is defined by the equation for free streaming,

$$
p^{\mu} \frac{\partial f}{\partial x^{\mu}}=0
$$

The solution of this equation describes the distribution function at any hypersurface $\tau=$ const by the use of the following substitution in the arguments of the function $f_{0}$ related to the initial proper time $\tau_{0}=1 \mathrm{fm} / \mathrm{c}$ :

$$
\begin{aligned}
& \mathbf{r}_{\mathrm{T}} \rightarrow \mathbf{r}_{\mathrm{T}}-\frac{\mathbf{p}_{\mathrm{T}}}{m_{\mathrm{T}}}\left(\tau \cosh \theta-\sqrt{\tau_{0}^{2}+\tau^{2} \sinh ^{2} \theta}\right), \\
& \theta \rightarrow \operatorname{arcsinh}\left(\frac{\tau}{\tau_{0}} \sinh \theta\right) .
\end{aligned}
$$

In what follows we will consider the properties of such a free-streaming expansion of a boost-invariant and cylindrically-symmetric finite system into vacuum as a first approximation, and discuss the possible whole picture of the early pre-thermal stage.

\section{COLLECTIVE VELOCITIES AND LOCAL ANISOTROPY IN PARTONIC SYSTEM}

Let us study the free-streaming stage of the evolution, supposing, as it was argued above, that the incoherent partonic system arises at the time of the order of $1 \mathrm{fm} / \mathrm{c}$ as a locally isotopic boost-invariant and weakly interacting gas. The gas 
will free stream into vacuum. The process itself will lead to a local anisotropy which we will study in this section. The increase of the anisotropy may be compensated by the process of turbulence (instability) and gradual thermalization associated with Balescu-Lenard term for QCD fields.

The analysis of the local anisotropy of the distribution function can be done in two ways. The first one deals with a study of distribution function properties, while the second one deals with the difference between spatial components of the energymomentum tensor in local rest frames. In both cases, we are forced to consider the distribution and the energy-momentum tensor in a co-moving reference frame determined by the collective velocity. Here we will apply both the Eckart and Landau-Lifshitz definitions of the collective velocities $\mathbf{v}(\mathbf{x})$ related to fairly small elements associated with the point $\left(x^{\mu}\right)$.

The connection between the global and local rest frame moving with the 3 -velocity $\mathbf{v}=\left(v^{i}\right)$, is Lorentz transformation defined by matrix of the form:

$$
\left(\Lambda_{v}^{\mu}\right)=\left(\begin{array}{c|c}
\gamma & v^{i} \gamma \\
\hline v^{j} \gamma & \delta^{i j}+v^{i} v^{j}(\gamma-1) / \mathbf{v}^{2}
\end{array}\right),
$$

where $\gamma=1 / \sqrt{1-v^{2}}$ is a Lorentz factor; $v \equiv|\mathbf{v}|$.

Making use of this matrix, the contravariant vector and tensor transformations read

$$
a^{\mu}=\Lambda^{\mu}{ }_{v} a_{*}^{v}, \quad a^{\mu \nu}=\Lambda_{\lambda}^{\mu} a_{*}^{\lambda \sigma} \Lambda_{\sigma}^{v}
$$

where $a_{*}^{\mu}$ and $a_{*}^{\mu \nu}$ denote these quantities in the co-moving reference frame.

Therefore the 3-vector $\mathbf{p}$ is transformed as follows

$$
\mathbf{p}=\mathbf{p}_{*}+\frac{\mathbf{v}}{v^{2}} \frac{\left(\mathbf{v} \mathbf{p}_{*}\right)\left(1-\sqrt{1-v^{2}}\right)+v^{2} E_{*}}{\sqrt{1-v^{2}}},
$$

where $E_{*}=\sqrt{m^{2}+\mathbf{p}_{*}^{2}}$.

It is possible to examine the anisotropy of the momentum distribution in different co-moving reference frames associated with different spacial points, where the 3-momentum $\mathbf{p}_{*}$ determines $\mathbf{p}$ in accordance with Eq. (8).

The local anisotropy reveals itself also in structure of the energy-momentum tensor, which in pseudo-Cartesian coordinates reads

$$
T^{\mu v}(x)=\int p^{\mu} p^{v} f(x, p) p_{\mathrm{T}} d p_{\mathrm{T}} d y d \phi
$$

where the Lorentz-invariant integration measure $d^{3} p / E$ in Cartesian variables is already re-written in Björken variables: $\left(p^{\mu}\right)=\left(m_{\mathrm{T}} \cosh y, p_{\mathrm{T}} \cos \phi, p_{\mathrm{T}} \sin \phi, m_{\mathrm{T}} \sinh y\right)$.

To find $T^{\mu v}$ in the central rapidity slice, we numerically calculate the energy-momentum tensor (9) at the longitudinal coordinate $z=0(\eta=0)$, when $\tau=t$. Due to symmetry properties of the distribution, one finds $T^{t z}=T^{x z}=T^{y z}=0$. Let $\psi$ be the angular direction relative to the radial axis $x$. Note that $T^{x y}=0$ at $\psi=n \pi / 2, n=0, \pm 1, \pm 2, \ldots$ Fixing $\psi=0$, the non-vanishing components of the energy-momentum tensor are

$$
\left(T^{\mu v}\right)=\left(\begin{array}{cccc}
T^{t t} & T^{t x} & 0 & 0 \\
T^{t x} & T^{x x} & 0 & 0 \\
0 & 0 & T^{y y} & 0 \\
0 & 0 & 0 & T^{z z}
\end{array}\right)
$$

It is understandable that the direction of collective velocities $\mathbf{v}$ in the global (origin) reference frame at $z=0$ should coincide with the vector $\mathbf{r}_{\mathrm{T}}$ and therefore $\mathbf{v}=(v \cos \psi, v \sin \psi, 0)$.

The tensor $T_{*}^{\mu \nu}$ in the co-moving reference frame, associated with the local velocity $\mathbf{v}$, is defined from (10) by use of the matrix $\Lambda_{\mu}{ }^{v}$ inverse to (6). (Actually, the matrix $\Lambda_{\mu}{ }^{v}$ is derived from (6) by replacement of $v^{i} \rightarrow-v^{i}$.) In the case of a boost, the components of the energy-momentum tensor in two reference frames are related by

$$
T_{*}^{\mu \nu}=\Lambda_{\lambda}^{\mu} T^{\lambda \sigma} \Lambda_{\sigma}{ }^{\nu}
$$

\section{A. Eckart Frame}

Now, we concentrate on the collective velocity computation. In this subsection we deal with 4 -velocity defined by Eckart:

$$
u_{\mathrm{E}}^{\mu}=\frac{N^{\mu}}{\sqrt{N_{\mathrm{v}} N^{v}}},
$$

where

$$
N^{\mu}=\int p^{\mu} f(x, p) p_{\mathrm{T}} d p_{\mathrm{T}} d y d \phi
$$

is the particle flux.

The collective 3-velocity is simply given by $\mathbf{v}_{\mathrm{E}}=\mathbf{u}^{\mathrm{E}} / u_{0}^{\mathrm{E}}$. The dependence of transverse velocity $v_{\mathrm{E}}=\sqrt{v_{x}^{2}+v_{y}^{2}}$ on $r_{\mathrm{T}}$ at $\eta=0$ is demonstrated in Fig. 3 .

Having numerically obtained the values of collective velocity, one can re-write the distribution function at the fixed point of space-time in the Eckart co-moving reference frame by means of a Lorentz transformation (8). At $\tau=\tau_{0}=1 \mathrm{fm} / \mathrm{c}$, the distribution is isotropic, as must be according to initial conditions. Increasing $\tau$, the distribution becomes more and more anisotropic that is reflected on the collective velocity development.

Another possibility to observe the anisotropy in the given system is to compare the components of energy-momentum tensor in a given co-moving reference frame, which is introduced by means of formula (11). The result of numerical calculations is shown in Fig. 4. Abbreviation "Arb. units" means that the distribution function is not normalized.

We find $T_{*}^{x x}=T_{*}^{y y}=T_{*}^{z z}$ at $\tau=\tau_{0}=1 \mathrm{fm} / \mathrm{c}$, that also confirms the isotropy at the initial moment. Changing $\tau$, the value of $T_{*}^{z z}$ turns out essentially less than $T_{*}^{x x}$ and $T_{*}^{y y}$, which also differ.

Remark that, putting $v=v_{\mathrm{E}}$ in Eq. (11), it is impossible to cancel $T_{*}^{t x}$ in whole region of values of $r_{\mathrm{T}}$. Further, we will demonstrate that the requirement $T_{*}^{t x}=0$ corresponds to definition of Landau-Lifshitz frame. 


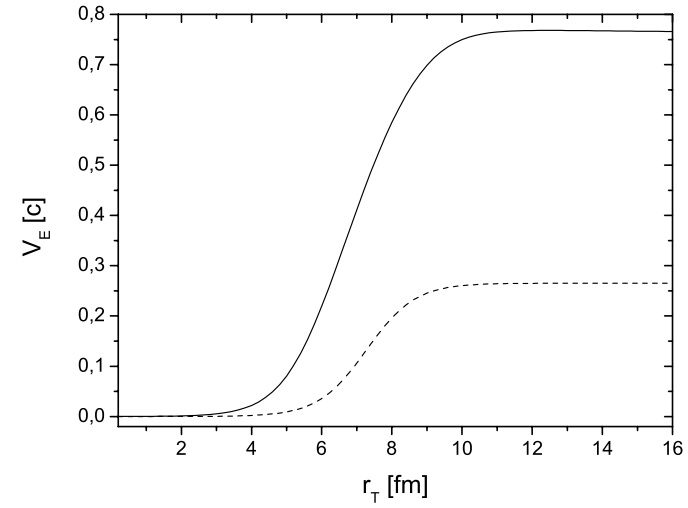

FIG. 3: The Eckart collective transverse velocity in a weakly interacting partonic system in the approximation of free streaming. The initial state at $1 \mathrm{fm} / \mathrm{c}$ is assumed to be quasi-thermal and corresponds to the distribution (2). Dashed curve correspond to $\tau=1.5 \mathrm{fm} / \mathrm{c}$, solid line $-3 \mathrm{fm} / \mathrm{c}$.

\section{B. Landau-Lifshitz Frame}

The Landau-Lifshitz definition of collective velocity can be formulated as

$$
u_{\mathrm{L}}^{\mu}=\frac{T^{\mu v} u_{v}^{\mathrm{L}}}{u_{\mathrm{L}}^{\lambda} T_{\lambda \sigma} u_{\mathrm{L}}^{\sigma}} .
$$

In general, this expression is equation with respect to $u_{\mathrm{L}}^{\mu}$, which should be solved numerically. However, in our case of cylindrical symmetry, when the free streaming is going on along $r_{\mathrm{T}}$-axis, the collective velocity can be found explicitly.

Substituting the expression for $T_{*}^{\mu \nu}$, the components of the collective 4-velocity in co-moving reference frame are

$$
\left(u_{* \mathrm{~L}}^{\mu}\right)=(1,0,0,0)=\left(1, \frac{T_{*}^{t x}}{T_{*}^{t t}}, 0,0\right) .
$$

It means that $T_{*}^{t x}=0$, and then one can obtain the expression for velocity in the global reference frame from Eq. (11):

$$
v_{\mathrm{L}}=\frac{T^{t t}+T^{x x}}{2 T^{t x}}\left(1-\sqrt{1-\frac{4\left(T^{t x}\right)^{2}}{\left(T^{t t}+T^{x x}\right)^{2}}}\right) .
$$

The behavior of $v_{\mathrm{L}}$ is shown in Fig. 5. The velocity $v_{\mathrm{L}}$ also vanishes at $\tau=\tau_{0}$. Although $v_{\mathrm{L}}$ is close to $v_{\mathrm{E}}$ (see Fig. 3) they are not completely coincided since the system is not in a locally equilibrated state.

In the case of $v=v_{\mathrm{L}}$ (see (11)), the anisotropy of the energymomentum tensor is demonstrated in Fig. 6 and it is qualitatively the same as in the Eckart case presented in Fig. 4.

\section{Analysis of Weak Anisotropy}

As one can see from Figs. 4 and 6, the diagonal spatial components of the energy-momentum tensor of the partonic

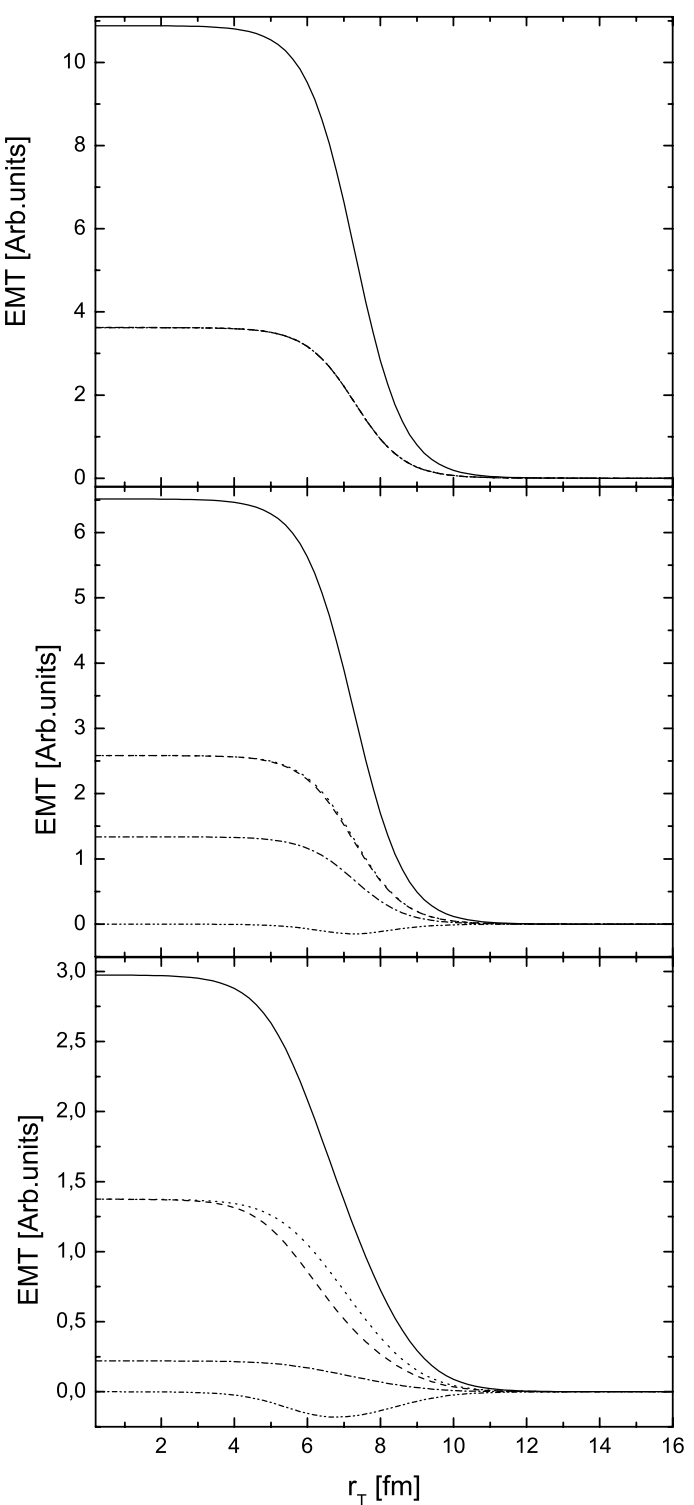

FIG. 4: The components of the energy-momentum tensor, $T_{*}^{t t}$ (solid), $T_{*}^{x x}$ (dashed), $T_{*}^{y y}$ (dotted), $T_{*}^{z z}$ (dot-dashed), $T_{*}^{t x}$ (dot-dot-dashed), at $\tau=1,1.5,3 \mathrm{fm} / \mathrm{c}$ (from above to below) and $\psi=0$. Eckart co-moving frame.

system, even if they were equal at the initial formation time [14], are splitting during free-streaming expansion so that $T_{*}^{y y}(x)>T_{*}^{x x}(x)>T_{*}^{z z}(x)$. Thus, the components of $T_{*}^{\mu v}(x)$ associated with directions of non-zero collective velocities (initial and developed) become suppressed as compare with other ones. Correspondingly, the particle distribution function gradually loses the local momentum isotropy during the expansion. Let us parameterize this anisotropy as depending on $\tau=t$ and $r_{\mathrm{T}}$ at fixed $z=0$.

It is useful to analyze the case of weak anisotropy and relate our result to other models. For this aim we represent the distribution function (2), (4), (5) in the form $f=F \cdot W$ where

$$
F(a)=\frac{1}{\exp \frac{a}{T}-1}, \quad W(b)=\frac{1}{\exp \frac{b-R}{\delta}+1} .
$$




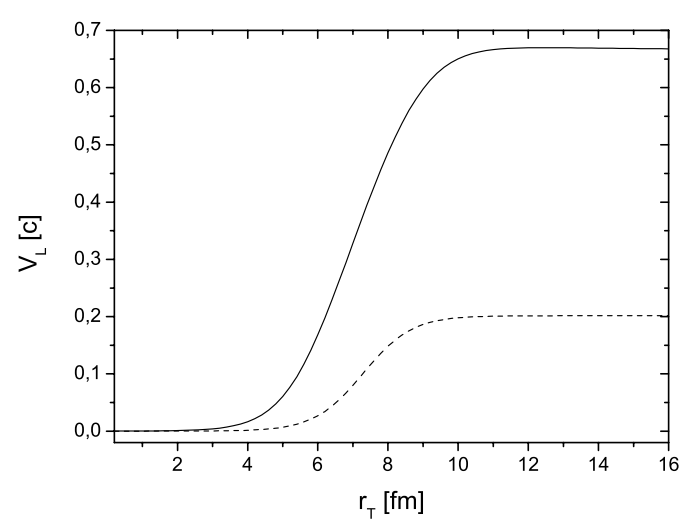

FIG. 5: The Landau-Lifshitz collective transverse velocity in weakly interacting partonic system in the approximation of free streaming. The initial state at $1 \mathrm{fm} / \mathrm{c}$ is supposed to be quasi-thermal and corresponds to the distribution (2). The dashed curve correspond to $\tau=1.5$ $\mathrm{fm} / \mathrm{c}$, solid line $-3 \mathrm{fm} / \mathrm{c}$.

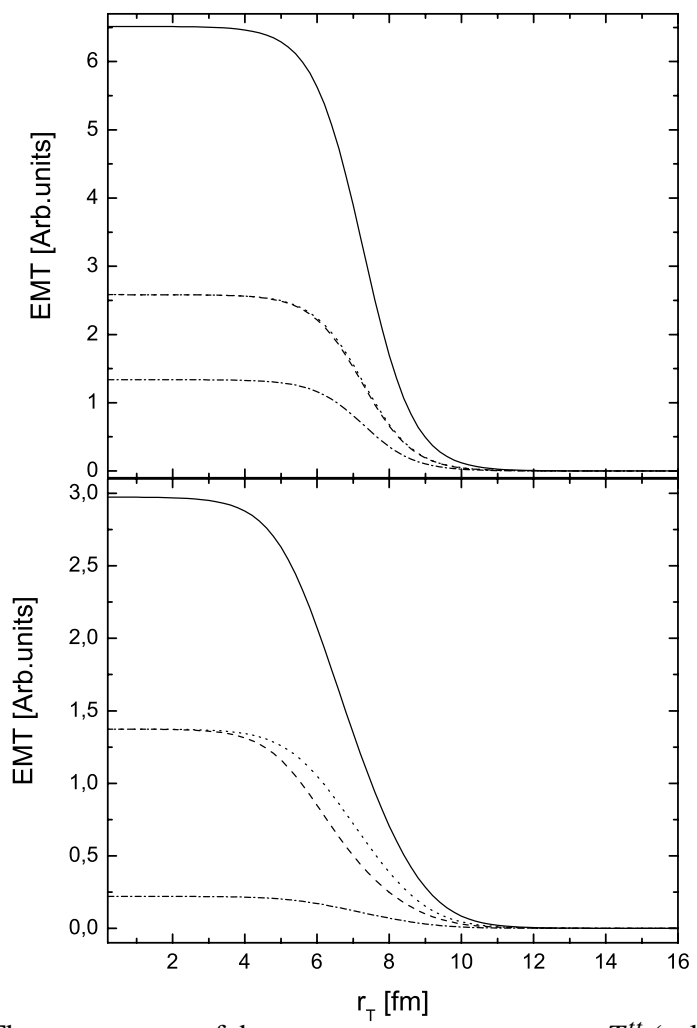

FIG. 6: The components of the energy-momentum tensor, $T_{*}^{t t}$ (solid), $T_{*}^{x x}$ (dashed), $T_{*}^{y y}$ (dotted), $T_{*}^{z z}$ (dot-dashed), at $\tau=1.5,3 \mathrm{fm} / \mathrm{c}$ (from above to below) and $\psi=0$. Landau-Lifshitz co-moving frame.

Fixing $z=0, \psi=0$, the arguments of these functions are presented as

$$
\begin{aligned}
a^{2}= & E^{2}+\xi p_{z}^{2} \\
b^{2}= & r_{\mathrm{T}}^{2}-2 \frac{r_{\mathrm{T}} p_{x}}{m^{2}+\mathbf{p}_{\mathrm{T}}^{2}} \tau_{0}(\sqrt{1+\xi} E-a) \\
& +\frac{\mathbf{p}_{\mathrm{T}}^{2}}{\left(m^{2}+\mathbf{p}_{\mathrm{T}}^{2}\right)^{2}} \tau_{0}^{2}(\sqrt{1+\xi} E-a)^{2},
\end{aligned}
$$

where we have introduced the parameter of anisotropy $\xi(\tau)=$ $\tau^{2} / \tau_{0}^{2}-1$ and $E=\sqrt{m^{2}+\mathbf{p}^{2}}$. Note that the same dependence of $\xi$ on the proper time has already pointed out in Ref.[15] to account for the longitudinally boost invariant expansion in partonic system.

Let us write the distribution function $f$ in the linear approximation in $\xi$. The form of such a distribution is

$$
\begin{aligned}
& f \approx f_{\text {iso }}+\frac{\xi(\tau)}{2}\left\{W\left(r_{\mathrm{T}}\right) \frac{d F(E)}{d E} \frac{p_{z}^{2}}{E}\right. \\
& \left.-F(E) \frac{d W\left(r_{\mathrm{T}}\right)}{d r_{\mathrm{T}}} \frac{p^{x} E \tau_{0}}{m^{2}+\mathbf{p}_{\mathrm{T}}^{2}}\left(1-\frac{p_{z}^{2}}{E^{2}}\right)\right\},
\end{aligned}
$$

where $f_{\text {iso }} \equiv F(E) W\left(r_{\mathrm{T}}\right)$ is the initial isotropic distribution function in the global reference frame.

We see that the first term in the brackets \{\} corresponds to the momentum anisotropy due to initial momentum inhomogeneity, while the second one is related to the initial inhomogeneity in coordinate space.

Since the axial symmetry we can put $\psi=0$, and the transition to the co-moving frame associated with some point $(x$, $y=0, z=0)$ is determined in the simple way:

$$
\begin{aligned}
& p^{x}=\frac{p_{*}^{x}+v E_{*}}{\sqrt{1-v^{2}}}, \quad E=\frac{E_{*}+v p_{*}^{x}}{\sqrt{1-v^{2}}}, \\
& p^{y}=p_{*}^{y}, \quad p^{z}=p_{*}^{z},
\end{aligned}
$$

where $E_{*}=\sqrt{m^{2}+\mathbf{p}_{*}^{2}}$.

Limiting ourselves by the linear approximation in the parameters of anisotropy, when $v$ is also assumed to be small and discarding the term of order $v \xi$, we find

$$
\begin{aligned}
& f_{*} \approx f_{\text {iso }}^{*}-W\left(r_{\mathrm{T}}\right) \frac{d F\left(E_{*}\right)}{d E^{*}} p_{*}^{x} v \\
& +\frac{\xi(\tau)}{2}\left\{W\left(r_{\mathrm{T}}\right) \frac{d F\left(E_{*}\right)}{d E_{*}} \frac{\left(p_{*}^{z}\right)^{2}}{E_{*}}\right. \\
& \left.-F\left(E_{*}\right) \frac{d W\left(r_{\mathrm{T}}\right)}{d r_{\mathrm{T}}} \frac{p_{*}^{x} E_{*} \tau_{0}}{m^{2}+\left(\mathbf{p}_{\mathrm{T}}^{*}\right)^{2}}\left[1-\left(\frac{p_{*}^{z}}{E_{*}}\right)^{2}\right]\right\},
\end{aligned}
$$

where $f_{\text {iso }}^{*} \equiv F\left(E_{*}\right) W\left(r_{\mathrm{T}}\right)$ is the isotropic distribution function in co-moving reference frame.

The radial collective velocity $v$ is actually a dependent parameter. Following the Eckart definition,

$$
v_{E}=\int p^{x} f(x, p) \frac{d^{3} p}{E} / \int f(x, p) d^{3} p
$$

(where we put $\psi=0$ again, and then the collective velocity direction coincides with $x$-axis), we obtain in the linear approximation

$$
v_{E} \approx \xi(\tau) \frac{\lambda_{0}}{\int f_{\text {iso }} d^{3} p}
$$

where

$$
\lambda_{n}=-\frac{\tau_{0}}{2} \int \frac{p_{x}^{2} E^{n} F(E)}{m^{2}+\mathbf{p}_{\mathrm{T}}^{2}}\left(1-\frac{p_{z}^{2}}{E^{2}}\right) \frac{d W\left(r_{\mathrm{T}}\right)}{d r_{\mathrm{T}}} d^{3} p .
$$


Similar computations can be also performed to obtain the form of Landau-Lifshitz collective velocity in the linear approximation in $\xi$. The result looks like

$$
v_{\mathrm{L}} \approx \xi(\tau) \frac{\lambda_{1}}{T_{t t}^{\text {iso }}+T_{x x}^{\text {iso }}},
$$

where $T_{t t}^{\text {iso }}$ and $T_{x x}^{\text {iso }}$ are the energy-momentum tensor components found on the basis of the isotropic distribution function $f_{\text {iso }} \equiv F(E) W\left(r_{\mathrm{T}}\right)$ in the global reference frame.

Now let us compare our results with the parametrization proposed by P. Romatschke with collaborators in Ref.[16]. It was assumed there that the anisotropic distribution function $h(\mathbf{p})$ is independent on space-time coordinates and constructed from an (arbitrary) isotropic distribution function by the rescaling of one direction in momentum space,

$$
h(\mathbf{p})=h_{\text {iso }}\left(\sqrt{\mathbf{p}^{2}+\xi(\mathbf{n p})^{2}}\right),
$$

where $\mathbf{n}$ is the direction of anisotropy, $\xi>-1$ is a constant parameter reflecting the strength of anisotropy. We omit here the normalization constant $N(\xi)$ which was used in Ref.[16] as not relevant to our problem since the particle number conservation during the evolution from initially isotropic state is guaranteed by Eq. (3).

Expanding the distribution $h(\mathbf{p})$, in the linear approximation in $\xi$ one can write that

$$
h(\mathbf{p}) \approx h_{\mathrm{iso}}(p)+\xi \frac{d h_{\mathrm{iso}}(p)}{d p} \frac{(\mathbf{n p})^{2}}{2 p} .
$$

It is easy to see that the expression (19) for the central slice $z=0, v_{z}=0$ is reduced to the last formula (28) as $\xi=\tau^{2} / \tau_{0}^{2}-1$, and the anisotropy vector $\mathbf{n}$ is directed along $z$ axis in the particular case assumed in Ref.[16], namely, a spatially homogeneous distribution, $W\left(r_{\mathrm{T}}\right) \equiv$ const, and massless particles, $E=\left|\mathbf{p}_{*}\right| \equiv p$.

In our inhomogeneous case, we can present a linearized form (22) for the distribution function in the local rest frame associated with some point $(x, y=0, z=0)$ as the following

$$
f_{*}\left(\tau, \mathbf{x}, \mathbf{p}_{*}\right) \approx f_{\text {iso }}^{*}\left(\mathbf{x},\left|\mathbf{p}_{*}\right|\right)+\xi(\tau) g\left(\mathbf{x}, \mathbf{p}_{*}\right) .
$$

\section{PROBLEM OF EVOLUTION AT PRE-THERMAL STAGE}

As it was demonstrated in $[15,16]$ the ansatz (27) is useful for analytical studies of the dispersion law and isotropization driven by instabilities. The latter can be caused by momentum anisotropy in a system of ultrarelativistic electro- or color- charged particles. The expression (29) also can be utilized for this aim. However, in our case, when initial the partonic system is supposed to be formed in a pseudo-thermal state due to Schwinger production in the pulse of chromoelectric field, the problem is to estimate whether this state, first, preserves during the evolution its (local) isotropy due to instability/turbulency mechanism and, then, if it transforms into

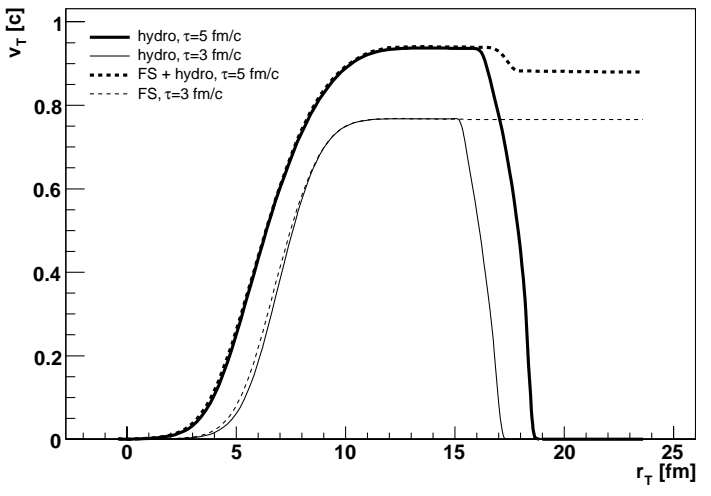

FIG. 7: The simulation of the transverse collective velocities (according to Eckart $\approx$ Landau-Lifshitz) of the quasi-free and almost massless partons within ideal hydrodynamics with the same initial conditions as for partonic system. The velocity is well approximated by such a hydro-evolution with extra-hard $\operatorname{EoS} P=0.45 \varepsilon$. The dashed curve corresponds to a weakly interacting partonic system at $\tau=3 \mathrm{fm} / \mathrm{c}$ and $5 \mathrm{fm} / \mathrm{c}$, solid line - to hydro-evolution at corresponding proper times.

a true thermal state due to the interactions. As we showed in previous section, the anisotropy caused by a free expansion of the finite system into vacuum can be characterized in the linear approximation by one parameter $\xi$, which is a function of the proper time $\tau$. One can estimate the possible rate of anisotropy growth at the following

$$
R(\tau) \equiv \frac{1}{f^{*}(\tau)} \frac{d f^{*}(\tau)}{d \tau} \simeq \frac{2 \tau}{\tau_{0}^{2}} \frac{g^{*}}{f_{\text {iso }}^{*}} .
$$

Approximate equality is written down for the case $\frac{\tau}{\tau_{0}}-1 \ll 1$.

In order to maintain the initial isotropization of the partonic system during the evolution, it is obvious that the rate $R(\tau)$ should be smaller than $1 / \tau_{\text {iso }}$, where $\tau_{\text {iso }}$ is a characteristic, or relaxation time of isotropization driven by instabilities. We plan to estimate $\tau_{\text {iso }}$ in a forthcoming work, as well as the rate of thermalization due to color interactions described by the Balescu-Lenard term in the kinetic equation.

In the previous section we analyzed the developing of collective transverse flow in the finite non-thermal partonic system in the first free-streaming approximation. The results are presented in Figs. 3 and 5. Unlike what was discussed in Sec. 3, the specific non-relativistic case, where the initial isotropy in the local rest frames of the distribution function is preserved during the further evolution, the ultrarelativistic free streaming expansion destroys local isotropy (and pseudo-thermalization), the distribution function and energy-momentum tensor become anisotropic in the local rest frames, and thus the development of the transverse velocities is not associated with hydrodynamics of an ultrarelativistic gas. Nevertheless, as we demonstrate in Fig. 7, such a development of transverse velocities can be approximated by the hydrodynamic expansion with abnormal hard EoS: $P=0.45 \varepsilon$ ("normal" upper limit $P=\varepsilon / 3$ has ultrarelativistic gas).

Therefore, it might be that a short thermalization time is not necessary for development of the observed radial flows. 
The flows can be developed, and even more effectively, at the pre-thermal or pseudo-thermal stage. The natural objection against such a scenario might mean cause a problem, not of radial, but of elliptic flows. They need earlier thermalization in order for the initial geometrical asymmetry in transverse plane to transform more effectively into momentum asymmetry. The pre-thermal transverse flows can smear out the asymmetry in momenta coming from the asymmetry in pressure gradients.

The solution of the problem could be to account for the residual - after the exclusion of the non-participants - of a transversely directed angular momentum which the system of participants has just after collision due to a shift of the center of masses of colliding nuclei in reaction plane, that is associated with impact parameter [17]. Then, as it is shown in Ref.[18], the corresponding tilt in the major axis of longitudinal expansion gives a positive contribution to the asymmetry of the particle momenta transverse to the beam plane, or in the $v_{2}$ coefficient. The account for an interplay between the initial pre-thermal transverse velocity and the angular momentum which the system of participants obtains in non-central collisions can open a new way in understanding the problem of matter evolution in nucleus-nucleus collisions.

\section{CONCLUSIONS}

The approximate conservation of the pion averaged phasespace density (APSD) in A+A collisions during the hadronic stage of the evolution allows one to explain the proportionality between the interferometry volume and different initial volumes, e.g., in non-central collisions, and also to explain the relative independence of the interferometry volumes on energy in central $\mathrm{Au}+\mathrm{Au}$ and $\mathrm{Pb}+\mathrm{Pb}$ collisions by an increase of transverse flow with energy. The hydrodynamic picture with initially non-zero transverse flow can help in describing the latter effect.
The other component of the RHIC HBT puzzle - the relatively small ratio of outward to sideward interferometry radii at protracted surface emission also needs an intense initial transverse flow for its explanation. The reason is that predominantly positive space-time $(r-t)$ correlations for emission points, which reduce the outward radius, can be realized only in hydrodynamic picture with strong enough transverse flow at initial moment.

We demonstrate here that the intensive transverse flows can be developed at the very early pre-thermal partonic stages when many hidden degrees of freedom, associated with incoherent partons, are liberated. It is shown that the development of the transverse velocities at the pre-thermal partonic stage can be approximated by the hydrodynamic expansion with abnormal hard equation of state. The interplay of those flows and angular momenta, which the system gets in noncentral collisions, could lead to a new scenario for the matter evolution and help to describe the experimental data in central and non-central A+A collisions.

\section{Acknowledgements}

Yu.S. would like to thanks very much to Tetsufumi Hirano who give possibility to use the hydrodynamic code to clarify some features of relativistic evolution.

The research described in this publication was made possible in part by Award No. UKP1-2613-KV-04 of the U.S. Civilian Research \& Development Foundation for the Independent States of the Former Soviet Union (CRDF). Also, the research carried out within the scope of the ERG (GDRE): Heavy ions at ultrarelativistic energies - a European Research Group comprising IN2P3/CNRS, EMN, Universite de Nantes, Warsaw University of Technology, JINR Dubna, ITEP Moscow and Bogolyubov Institute for Theoretical Physics NAS of Ukraine.
[1] C. Adler et al. (STAR Collaboration), Phys. Rev. Lett. 87, 082301 (2001); K. Adcox et al. (PHENIX Collaboration), Phys. Rev. Lett. 88, 192302 (2002); S. Kniege et al. (NA49 Collaboration), J. Phys. G 30, S1073 (2004).

[2] U. Heinz, P.F. Kolb, arXiv:hep-ph/0204061; U. Heinz, Nucl. Phys. A 721, 30 (2003); S. Pratt, Nucl. Phys. A 715, 389c (2003); S. Soff, S. Bass, D. Hardtke, and S. Panitkin, Nucl. Phys. A 715, 801c (2003).

[3] S.V. Akkelin, Yu.M. Sinyukov, Phys. Rev. C 70, 064901 (2004).

[4] S.V. Akkelin, Yu.M. Sinyukov, Phys. Rev. C 73, 034908 (2006); Nucl. Phys. A 774, 674 (2006).

[5] H. Appelshauser, J. Phys. G 30, S935 (2004).

[6] M.S. Borysova, Yu.M. Sinyukov, S.V. Akkelin, B. Erazmus, and Iu.A. Karpenko, Phys. Rev. C 73, 024903 (2006).

[7] Iu.A. Karpenko, Master Thesis, National University of Kiev (2006).

[8] Yu.M. Sinyukov, Iu.A. Karpenko, Acta Phys. Hung. A 25, 141 (2006).

[17] Yu.M. Sinyukov, Act. Phys. Polon. B 37, 3343 (2006).
[10] S. Mashkevich, private communication

[11] L. McLerran and R. Venugopalan, Phys. Rev. D 49, 2233 (1994); ibid, 3352 (1994); ibid D 50, 2225 (1994); ibid D 53, 458 (1996); ibid D 59, 09400 (1999).

[12] St. Mrowczynski, Phys. Rev. D 39, 1940 (1989).

[13] A. Krasnitz, Y. Nara, and R. Venugopalan, Nucl. Phys. A 727, 427 (2003) [arXiv:hep-ph/0305112].

[14] D. Kharzeev, E. Levin, and K. Tuchin, arXiv:hep-ph/0602063.

[15] P. Romatschke and A. Rebhan, Phys. Rev. Lett. 97, 252301 (2006) [arXiv:hep-ph/0605064].

[16] P. Romatschke and M. Strickland, Phys. Rev. D 70, 116006 (2004) [arXiv:hep-ph/0406188]; P. Romatschke and M. Strickland, Phys. Rev. D 68, 036004 (2003) [arXiv:hep-ph/0304092]; P. Romatschke, Ph.D. Dissertation, arXiv:hep-ph/0312152.

[17] Yu.M. Sinyukov, Act. Phys. Polon. B 37, 3343 (2006).

[18] T. Csörgố, S.V. Akkelin, Y. Hama, B. Lukacs, and Yu.M. Sinyukov, Phys. Rev. C 67, 034904 (2003). 